\title{
Small cell transformation of non-small cell lung cancer on immune checkpoint inhibitors: uncommon or under- recognized?
}

\author{
Kartik Sehgal (D , ${ }^{1}$ Andreas Varkaris, ${ }^{1}$ Hollis Viray, ${ }^{1}$ Paul A VanderLaan, ${ }^{2}$ \\ Deepa Rangachari, ${ }^{1}$ Daniel B Costa ${ }^{1}$
}

To cite: Sehgal K, Varkaris A, Viray $\mathrm{H}$, et al. Small cell transformation of nonsmall cell lung cancer on immune checkpoint inhibitors: uncommon or under-recognized? Journal for ImmunoTherapy of Cancer 2020;8:e00697. doi:10.1136/ jitc-2020-000697

Accepted 19 May 2020
Check for updates

(C) Author(s) (or their employer(s)) 2020. Re-use permitted under CC BY-NC. No commercial re-use. See rights and permissions. Published by BMJ.

${ }^{1}$ Medical Oncology, Beth Israel Deaconess Medical Center, Boston, Massachusetts, USA ${ }^{2}$ Pathology, Beth Israel Deaconess Medical Center, Boston, Massachusetts, USA

\section{Correspondence to}

Dr Kartik Sehgal, Division of Medical Oncology, Department of Medicine, Beth Israel Deaconess Medical Center, Boston, Massachusetts, United States;

ksehgal@bidmc.harvard.edu

Dr Daniel B Costa, Division of Medical Oncology, Department of Medicine, Beth Israel Deaconess Medical Center, Boston, Massachusetts, United States;

dbcosta@bidmc.harvard.edu

\section{ABSTRACT}

Background Histological transformation of oncogenedriven lung adenocarcinoma to small cell lung cancer (SCLC) following treatment with tyrosine kinase inhibitors (TKIs) is a well-described phenomenon. Whether a similar transformation may drive acquired resistance to immune checkpoint inhibitors (ICPIs) in non-SCLC (NSCLC) is uncertain. Hence, tissue biopsies are not universally recommended at progression of NSCLC on ICPIs, unlike TKIs.

Case presentation We report a case of a woman in her mid-60s with a 35 pack-years tobacco history and stage IV squamous cell lung carcinoma with no targetable genomic alterations, whose disease progressed within 4 months of first line carboplatin/gemcitabine therapy. Her treatment was switched to second line nivolumab monotherapy which resulted in sustained partial response lasting 21 months. She subsequently developed rapid, bulky progression of mediastinal disease. Biopsy showed transformation to SCLC. Comparison of genomic profiling results from the initial NSCLC diagnosis and SCLC transformation revealed near-identical tumor profiles. Her disease responded to next line carboplatin/etoposide, though lasting for only 10 months. She died 14 months after detection of neuroendocrine transformation of her NSCLC.

Systematic review We performed a systematic review of the literature to identify similar cases of NSCLC-to-small cell transformation on ICPIs. Nine patients, including our index case, were identified, with seven $(77.8 \%)$ on nivolumab and two $(22.2 \%)$ on pembrolizumab monotherapy. Median survival time since small cell transformation was 13.0 months $(95 \% \mathrm{Cl}$ 2.0 to 16.0). Using our patient case as a framework, we further discuss the lack of consensus criteria to distinguish small cell transformation from de novo metachronous SCLC.

Conclusions Histological transformation to SCLC is a potential mechanism of acquired resistance to ICPIs in NSCLC. Repeat tissue biopsies should be considered at the time of progression, similar to oncogene-directed therapies. Prospective larger studies are warranted to further characterize NSCLC-to-small cell transformation on ICPIs using molecular fingerprinting with paired tumor genomic profiles, evaluation of neuroendocrine features at baseline and consideration of initial response.

\section{BACKGROUND}

Resistance to tyrosine kinase inhibitors (TKIs) in epidermal growth factor receptor (EGFR) mutated non-small cell lung cancer (NSCLC) is well established to be mediated by histological transformation to SCLC in 3\%-14\% of cases. ${ }^{1-3}$ Similarly, transformation of prostate adenocarcinoma to small cell carcinoma on androgen-deprivation therapy is reported to occur at an incidence of $17 \%$ and is associated with poor survival outcomes. ${ }^{45}$ More recently, reports have emerged regarding SCLC transformation of NSCLC as a resistance mechanism to immune checkpoint inhibitors (ICPIs). However, unlike disease progression on TKIs, repeat tissue biopsies are not universally recommended at the time of NSCLC progression on ICPIs.

\section{CASE PRESENTATION}

In our practice, we cared for a patient who had small cell transformation of stage IV poorly differentiated squamous cell carcinoma of the lung after prolonged nivolumab monotherapy (figure 1). She was in her mid60 s with a history of 35 pack-years of smoking at the time of diagnosis of her lung cancer (metastatic to lungs, mediastinal lymph nodes and L1 vertebral body) with no targetable genomic alterations. After a short-lived response to first-line platinum-gemcitabine chemotherapy lasting less than 4 months, she had progression of her disease. She was then switched to nivolumab monotherapy, with sustained partial response for 21 months. On follow-up imaging, she was noted to have bulky mediastinal and right hilar lymphadenopathy; biopsy showed SCLC. Review of the biopsy at initial NSCLC diagnosis did not show any small cell component. Tumor genomic profiling performed at initial 


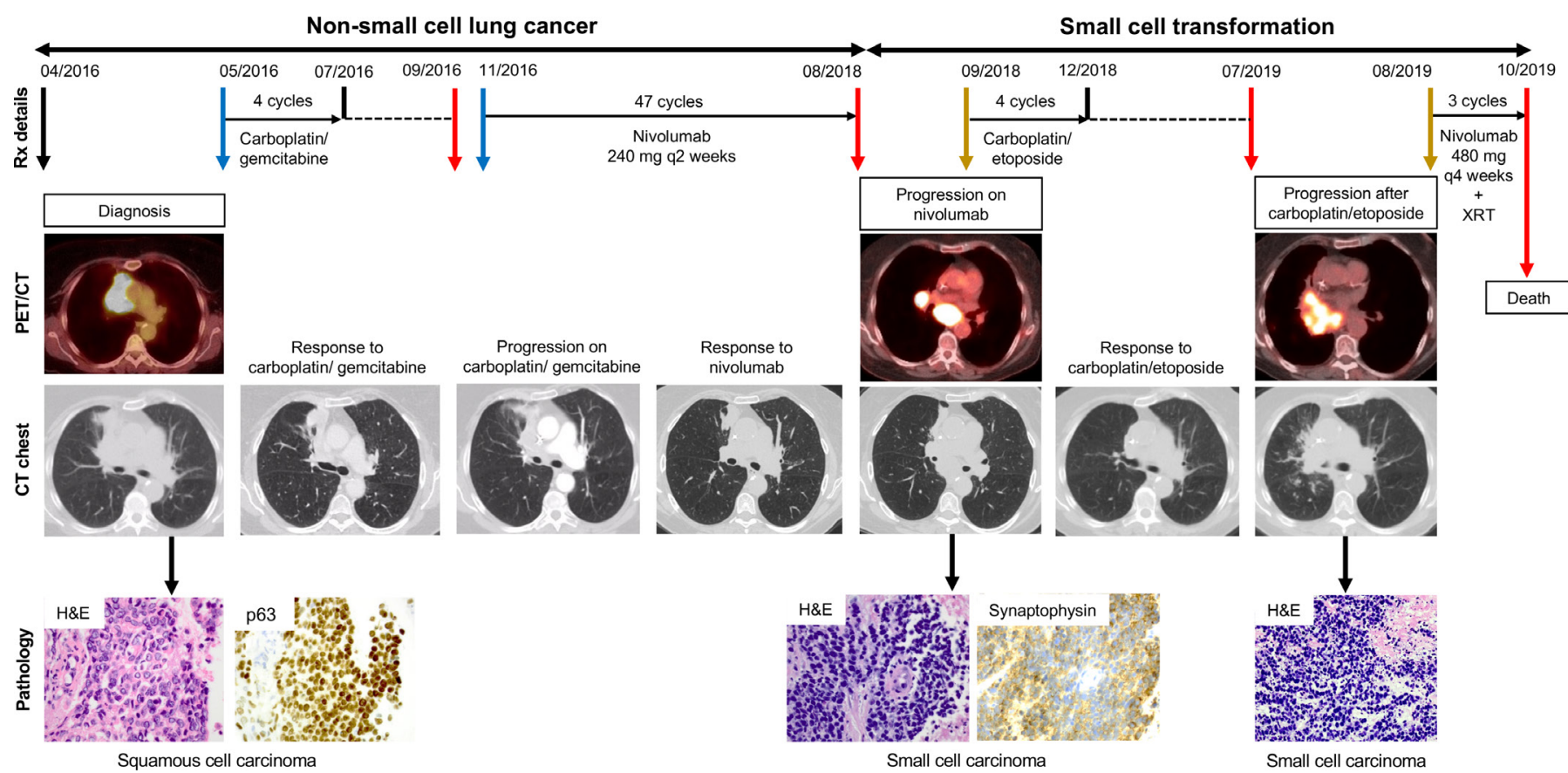

Figure 1 Case presentation of small cell transformation of non-small cell lung cancer on nivolumab monotherapy, including treatment details, and radiographic and pathological findings. Time on therapy is not drawn to scale. CT, computed tomography; H\&E, hematoxylin and eosin; PET, positron emission tomography; Rx, treatment; XRT, radiotherapy.

diagnosis and following disease progression on nivolumab showed nearly identical results (table 1). Treatment with carboplatin/etoposide led to near-complete response, however, lasting for only 10 months. Biopsy of the tumor again confirmed small cell histology. She was treated with concurrent nivolumab and radiotherapy to the chest, though ultimately elected to pursue comfort focused care and died 14 months after the detection of neuroendocrine transformation.

\section{SYSTEMATIC REVIEW}

We performed a systematic review of the literature, in accordance with Preferred Reporting Items for Systematic Reviews and Meta-analyses guidelines, to identify similar published reports of NSCLC-to-small cell transformation on ICPIs (figure 2). We searched PubMed, Embase and the American Society of Clinical Oncology/International Association for the Study of Lung Cancer virtual meeting library databases on 7 December 2019, using the keywords small cell transformation/neuroendocrine transformation with or without ICPIs/anti-PD-1/pembrolizumab/ nivolumab/atezolizumab/durvalumab. Two investigators (KS and $\mathrm{AV}$ ) independently reviewed abstracts and full-text articles. Patients with advanced NSCLC who had received molecularly targeted therapies prior to small cell transformation or non-lung primary cancers were excluded. Nine patients were identified from five articles (three case series ${ }^{6-8}$ and two case reports ${ }^{9}{ }^{10}$ ) and one meeting abstract (index case).

All patients were on treatment with ICPIs at the time of detection of SCLC, with seven $(77.8 \%)$ on nivolumab and two $(22.22 \%)$ on pembrolizumab monotherapy.
Five $(55.6 \%)$ were male; median age was 68 years (range $65-75$ years). All eight $(100 \%)$ patients for whom smoking history was described had history of tobacco exposure. The median number of treatments received before ICPI was 1 (range 0-3). All (100\%) patients had received chemotherapy before switch to either second line or maintenance ICPI. After detection of small cell transformation, seven $(77.8 \%)$ patients received carboplatin/etoposide as the next immediate line of therapy. Among eight patients for whom survival data was available, median survival since detection of small cell transformation was 13.0 months (95\% CI 2.0 to 16.0 months; Stata/IC V.15.1), which was comparable to 10.9 months (95\% CI 8.0 to 13.7 months) previously reported with transformed EGFR-mutant lung adenocarcinoma on TKIs. ${ }^{11}$ The full clinicopathological and tumor genomic details of these cases are summarized in table 1 .

\section{DISCUSSION AND CONCLUSIONS}

No consensus guidelines exist on how to define NSCLCto-small cell transformation and distinguish it from new primary SCLC. Absence of neuroendocrine features on initial biopsy, protracted response to nivolumab monotherapy and the near-identical genomic profile of the two tumors favored the diagnosis of histological transformation in our patient. Proof of transformation with molecular fingerprinting was described in only two of the other eight patients (table 1 ). The genomic profiles of 'transformed small cell tumors' in three patients were completely different from the 'original' NSCLC tumors, which raises the question of true treatmentinduced transformation versus metachronous primary 


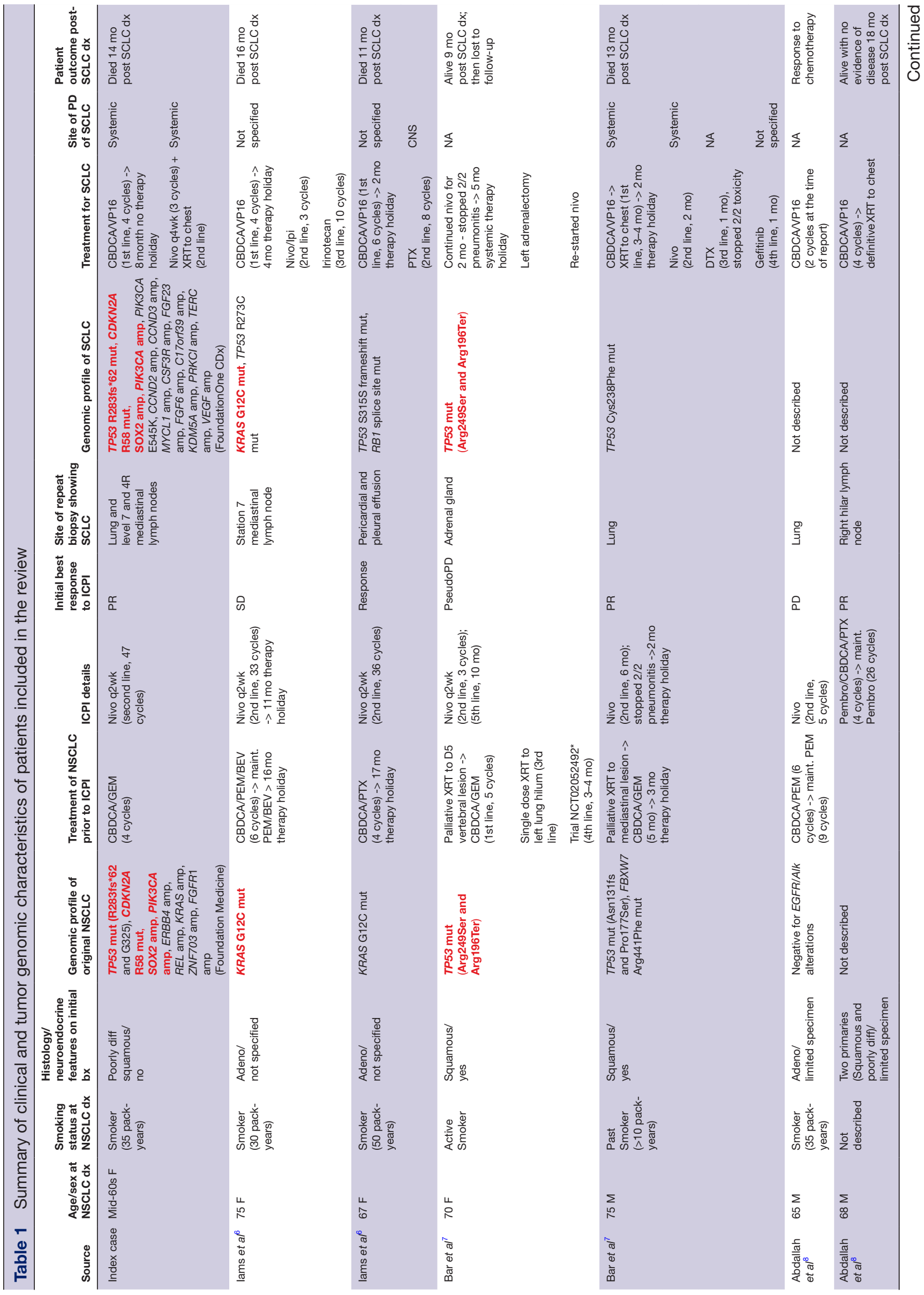



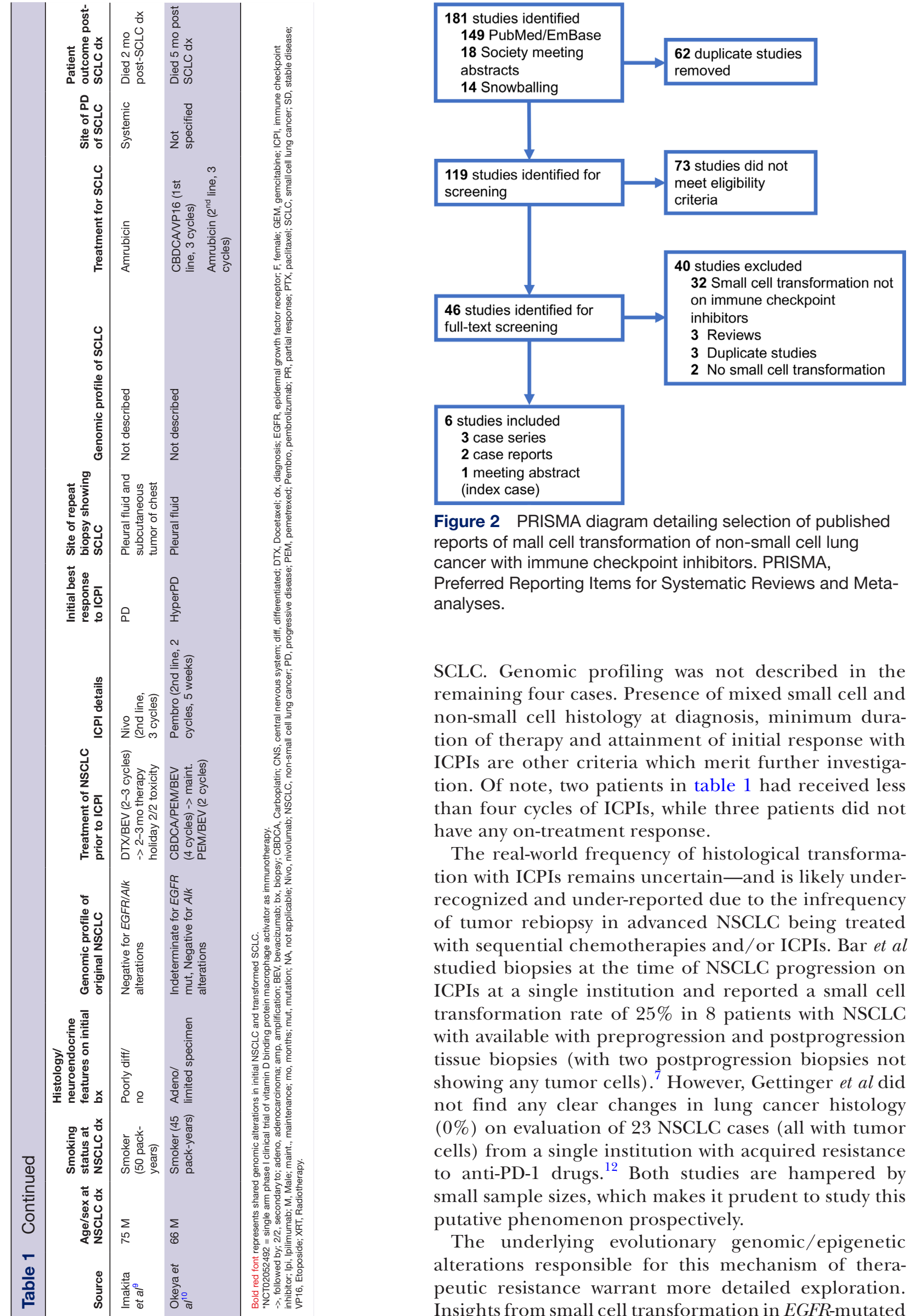

Figure 2 PRISMA diagram detailing selection of published reports of mall cell transformation of non-small cell lung cancer with immune checkpoint inhibitors. PRISMA, Preferred Reporting Items for Systematic Reviews and Metaanalyses.

SCLC. Genomic profiling was not described in the remaining four cases. Presence of mixed small cell and non-small cell histology at diagnosis, minimum duration of therapy and attainment of initial response with ICPIs are other criteria which merit further investigation. Of note, two patients in table 1 had received less than four cycles of ICPIs, while three patients did not have any on-treatment response.

The real-world frequency of histological transformation with ICPIs remains uncertain-and is likely underrecognized and under-reported due to the infrequency of tumor rebiopsy in advanced NSCLC being treated with sequential chemotherapies and/or ICPIs. Bar et al studied biopsies at the time of NSCLC progression on ICPIs at a single institution and reported a small cell transformation rate of $25 \%$ in 8 patients with NSCLC with available with preprogression and postprogression tissue biopsies (with two postprogression biopsies not showing any tumor cells). ${ }^{7}$ However, Gettinger et al did not find any clear changes in lung cancer histology $(0 \%)$ on evaluation of 23 NSCLC cases (all with tumor cells) from a single institution with acquired resistance to anti-PD-1 drugs. ${ }^{12}$ Both studies are hampered by small sample sizes, which makes it prudent to study this putative phenomenon prospectively.

The underlying evolutionary genomic/epigenetic alterations responsible for this mechanism of therapeutic resistance warrant more detailed exploration. Insights from small cell transformation in EGFR-mutated 
lung adenocarcinoma and prostate adenocarcinoma may help direct further mechanistic investigations towards study of common cell-of-origin, drug-tolerant persister state and stromal interactions. ${ }^{2}{ }^{13-15}$ In the meanwhile, we recommend that tissue biopsies should be considered at the time of NSCLC progression on ICPIs similar to TKIs, if safe and feasible from the patient perspective.

\section{Twitter Kartik Sehgal @KartikSehgal_MD}

Acknowledgements We acknowledge the support of team at Foundation Medicine Inc. for report of tumor genomic findings.

Contributors KS and DBC conceptualized and designed the study. KS and AV independently screened all the studies. All authors participated in the acquisition, analysis or interpretation of data, and in the drafting, critical revision, and approval of final version of the manuscript.

Funding This work was funded in part by the National Institutes of Health (NIH)/ National Cancer Institute (NCI) (grant R37CA218707 awarded to DBC).

Disclaimer The funders/sponsors had no role in the design and conduct of the study; collection, management, analysis, and interpretation of the data; preparation, review, or approval of the manuscript; and decision to submit the manuscript for publication. The content is solely the responsibility of the authors and does not necessarily represent the official views of the National Institutes of Health.

Competing interests PAVL reports personal fees (consulting fees and honoraria) from Gala Therapeutics, Flatiron Health, Caris Life Sciences and Foundation Medicine; all outside the submitted work. DR reports non-financial support (institutional research support) from Bristol-Myers Squibb, Novocure, and Abbvie/Stemcentrx, all outside the submitted work. DBC reports personal fees (consulting fees and honoraria) and nonfinancial support (institutional research support) from Takeda/Millennium Pharmaceuticals, and AstraZeneca, and Pfizer, as well as nonfinancial support (institutional research support) from Merck Sharp and Dohme Corporation, Merrimack Pharmaceuticals, Bristol-Myers Squibb, Clovis Oncology, Spectrum Pharmaceuticals and Tesaro, all outside the submitted work.

\section{Patient consent for publication Not required.}

Ethics approval Patient information was collected from medical records in accordance with research protocols approved by the Beth Israel Deaconess Medical Center and Dana-Farber/Harvard Cancer Center institutional review boards.

Provenance and peer review Not commissioned; externally peer reviewed.

Open access This is an open access article distributed in accordance with the Creative Commons Attribution Non Commercial (CC BY-NC 4.0) license, which permits others to distribute, remix, adapt, build upon this work non-commercially, and license their derivative works on different terms, provided the original work is properly cited, appropriate credit is given, any changes made indicated, and the use is non-commercial. See http://creativecommons.org/licenses/by-nc/4.0/.
ORCID iD

Kartik Sehgal http://orcid.org/0000-0003-4391-6943

\section{REFERENCES}

1 Sequist LV, Waltman BA, Dias-Santagata D, et al. Genotypic and histological evolution of lung cancers acquiring resistance to EGFR inhibitors. Sci Transl Med 2011;3:75ra26.

2 Oser MG, Niederst MJ, Sequist LV, et al. Transformation from nonsmall-cell lung cancer to small-cell lung cancer: molecular drivers and cells of origin. Lancet Oncol 2015;16:e165-72.

3 Farago AF, Piotrowska Z, Sequist LV. Unlocking the mystery of smallcell lung cancer transformations in EGFR mutant adenocarcinoma. $J$ Clin Oncol 2017;35:2987-8.

4 Aggarwal R, Huang J, Alumkal JJ, et al. Clinical and genomic characterization of Treatment-Emergent small-cell neuroendocrine prostate cancer: a multi-institutional prospective study. J Clin Oncol 2018;36:2492-503.

5 Volta AD, Cosentini D, Antonelli A, et al. Transformation of prostate adenocarcinoma into small-cell neuroendocrine cancer under androgen deprivation therapy: much is achieved but more information is needed. J Clin Oncol 2019;37:350-1.

6 lams WT, Beckermann KE, Almodovar K, et al. Small cell lung cancer transformation as a mechanism of resistance to PD-1 therapy in KRAS-mutant lung adenocarcinoma: a report of two cases. $J$ Thorac Oncol 2019;14:e45-8.

7 Bar J, Ofek E, Barshack I, et al. Transformation to small cell lung cancer as a mechanism of resistance to immunotherapy in non-small cell lung cancer. Lung Cancer 2019;138:109-15.

8 Abdallah N, Nagasaka M, Abdulfatah E, et al. Non-Small cell to smal cell lung cancer on PD-1 inhibitors: two cases on potential histologic transformation. Lung Cancer 2018;9:85-90.

9 Imakita T, Fujita K, Kanai O, et al. Small cell lung cancer transformation during immunotherapy with nivolumab: a case report. Respir Med Case Rep 2017;21:52-5.

10 Okeya K, Kawagishi Y, Muranaka E, et al. Hyperprogressive disease in lung cancer with transformation of adenocarcinoma to small-cell carcinoma during pembrolizumab therapy. Intern Med 2019;58:3295-8.

11 Marcoux N, Gettinger SN, O'Kane G, et al. Egfr-Mutant adenocarcinomas that transform to small-cell lung cancer and other neuroendocrine carcinomas: clinical outcomes. J Clin Oncol 2019;37:278-85.

12 Gettinger SN, Wurtz A, Goldberg SB, et al. Clinical features and management of acquired resistance to PD-1 axis inhibitors in 26 patients with advanced non-small cell lung cancer. $J$ Thorac Oncol 2018;13:831-9.

13 Lee J-K, Lee J, Kim S, et al. Clonal history and genetic predictors of transformation into small-cell carcinomas from lung adenocarcinomas. J Clin Oncol 2017;35:3065-74.

14 Mishra R, Haldar S, Placencio V, et al. Stromal epigenetic alterations drive metabolic and neuroendocrine prostate cancer reprogramming. J Clin Invest 2018;128:4472-84.

15 Beltran H, Hruszkewycz A, Scher HI, et al. The role of lineage plasticity in prostate cancer therapy resistance. Clin Cancer Res 2019;25:clincanres.1423.2019-6924. 